\title{
FORMACIÓN PARA LA INFORMACIÓN: INFORMACIÓN PARA LA INNOVACIÓN
}

\section{Noel Angulo}

\section{Resumen:}

Se aborda el concepto de búsqueda de información y sus alcances, así como las herramientas comunes a la búsqueda de información en Internet; se describen los diferentes tipos de búsqueda, los diferentes tipos de buscadores de información así como las técnicas básicas de información en Internet. Se asume que los docentes interesados en la innovación educativa tendrán mejores posibilidades de éxito si se ocupan de mejorar sus competencias en la búsqueda, recuperación, registro y organización de información y su conversión en conocimiento.

\section{Palabras clave:}

Búsqueda de información; Recuperación de información. Información para la innovación; Competencia en información; Herramientas de búsqueda

\section{TRAINING INFORMATION: INFORMATION FOR INNOVATION}

\begin{abstract}
:
It's presented the information search concept and its reaches, as well as the common tools to the information search in Internet; the different search types are described, and the different types of information search tools and the basic techniques of information search in Internet. It is assumed that the professors interested in the educational innovation will have better possibilities of success if they have competencies in the search, retrieval, registration and organization of information and its conversion in knowledge.
\end{abstract}

\section{Keywords:}

Information search; Information retrieval; Information for innovation; Information competencies; Search tools 


\section{Introducción}

La Edad de Piedra se caracterizó por el uso inteligente de herramientas toscas. La Era de la Información se ha caracterizado, al menos hasta ahora, por el uso tosco de herramientas inteligentes. Anónimo.

La búsqueda de información es hoy en día el modelo de navegación más común en la Red. La búsqueda parece cada vez más sencilla, basta anotar una o más palabras en una caja de texto de una herramienta de búsqueda y en respuesta se obtiene la información que nos puede ayudar a lograr nuestros objetivos o simplificar nuestro trabajo. Las herramientas de búsqueda disponibles en Internet han potenciado nuestra capacidad para relacionar necesidades y propósitos con los recursos de información que pueden contribuir a su satisfacción, no obstante la facilidad de operación nos hace olvidar el ejercicio intelectual de pensar para precisar lo que realmente necesitamos o de expresarlo en una forma adecuada para delegar la búsqueda al sistema que estemos utilizando, esto puede transformar la experiencia del usuario en una frustración o en una falsa idea de que la información que necesita no existe en la Red.

Es frecuente pensar que en Internet no se requiere formación alguna para localizar la información, no obstante, siempre tendremos mejores resultados si dedicamos un tiempo para conocer las herramientas de búsqueda y su forma de operación, sobre todo cuando se desea obtener información realmente útil y actualizada, además de tener la seguridad en cuanto a su calidad y confiabilidad. Observando el desempeño de los usuarios, se puede apreciar que, ya sea por descuido o por desconocimiento, se hace uso limitado de las herramientas de búsqueda, el usuario común sólo emplea unas pocas palabras para construir su argumento de búsqueda para después seleccionar entre los resultados obtenidos la información que consideran de utilidad.

Las fallas en la recuperación de información se deben, en algunos casos, a una inadecuada selección de las palabras o a una deficiente combinación de éstas, al momento de construir el argumento de búsqueda, lo que puede provocar que se descarten involuntariamente los documentos pertinentes a las necesidades, de tal manera que resulta imposible encontrar lo que estamos buscando, pese a que esté disponible en la Red. A este fenómeno se le llama silencio. En otras ocasiones, las palabras utilizadas en la operación de búsqueda y su combinación propician que la búsqueda arroje una cantidad excesiva de documentos, los cuales pueden tener poca o ninguna relación con lo que 
realmente se busca, a este fenómeno se le llama ruido. En ambos casos se trata de problemas que se pueden resolver con el desarrollo de la competencia en información.

Pese a que generalmente se da poca atención a la formación para la búsqueda de información, el volumen de información ${ }^{1}$ disponible en la Red crece de manera continua y su impacto social es cada vez mayor, razón por lo que ahora es difícil prescindir de las habilidades para encontrar datos en Internet, sobre todo cuando no se encuentran disponibles en ningún otro lugar, además de que ofrece la posibilidad de acceder de manera inmediata a información sin importar su ubicación física. Por otra parte no podemos seguir pensando en un nivel estándar de conocimiento al que los estudiantes deben acceder. La explosión de la información, y el nivel de obsolescencia del conocimiento nos obligan a la renovación constante, por lo que en lugar de atender los conocimientos declarativos para la formación de las nuevas generaciones, debemos preocuparnos si están preparados para autoformarse y acceder a la información cuando la necesiten (FUENTES, 2001).

\section{Búsqueda de información}

La búsqueda y recuperación de información podemos entenderla como el conjunto de tareas destinadas a localizar y acceder a los recursos de información pertinentes a nuestras necesidades y propósitos. Lo anterior hace evidente la importancia de desarrollar las habilidades para identificar y representar las necesidades de información, además de las habilidades en la construcción y aplicación de estrategias de búsqueda, (véase figura 1), no obstante, de acuerdo con Ricardo Fornás, (citado por Tomás Delclós 2007), el promedio de consultas en Internet se hace con sólo tres palabras, lo que significa que una mayoría de los usuarios desconoce los métodos para acotar una búsqueda, ignora lo que es una búsqueda booleana y mucho menos están habilitada para diseñar y aplicar una estrategia destinada a obtener la mayor ventaja de las herramientas de búsqueda y de los recursos de información disponibles en la red. 


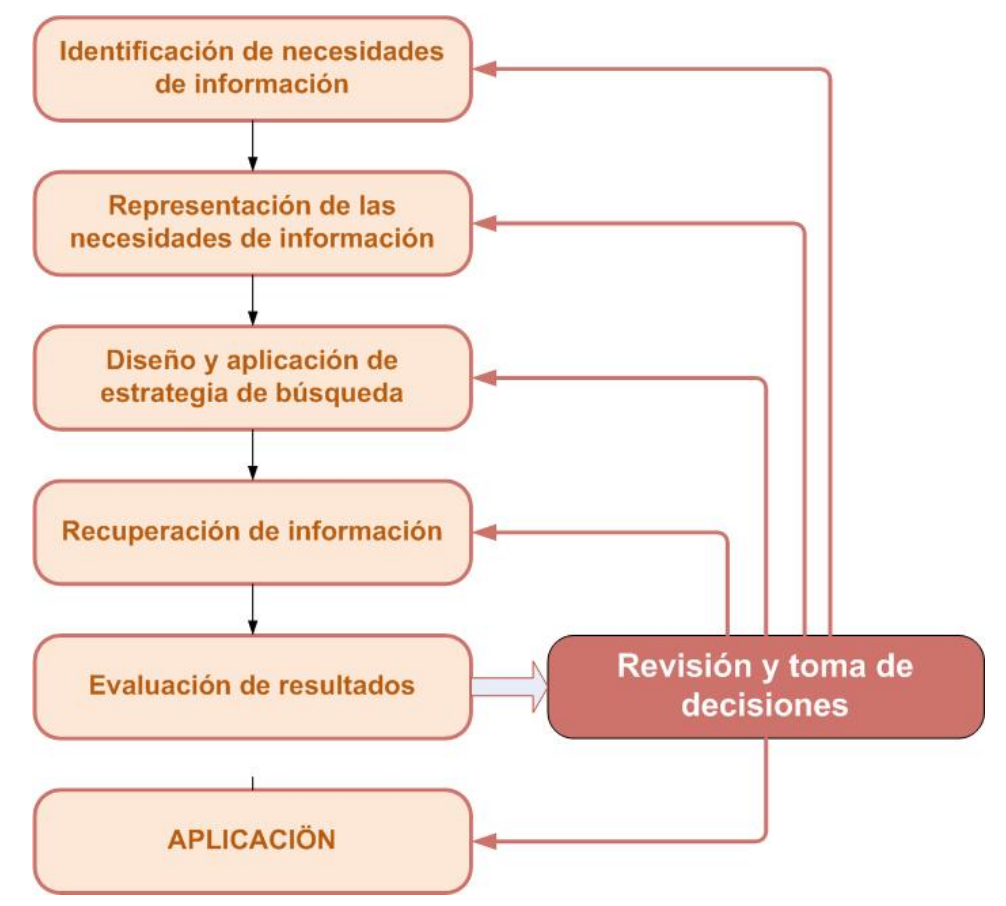

Figura 1 Búsqueda de información

\section{Estrategia de Búsqueda}

La clave en la búsqueda exitosa de información radica, principalmente, en cómo percibimos, interpretamos y representamos nuestras necesidades de información para expresarlas en una proposición, o argumento de búsqueda en la base de datos. Y desde luego también es importante tener una idea previa de las herramientas de búsqueda que debemos emplear, dependiendo del contexto de aplicación.

Una vez planteada la necesidad de información se debe desarrollar una estrategia de búsqueda. Ésta debe incluir las fuentes de información que se van a utilizar, y como cada una de las fuentes de información tiene sus características, la selección de éstas dependerá de las necesidades de información y de la disponibilidad de acceso. El diseño de la estrategia inicia con la definición de los términos o palabras claves, que mejor expresen la necesidad de información. La construcción de una estrategia de búsqueda es un ejercicio interactivo, donde se va refinando el perfil de búsqueda, dentro del proceso mismo de recuperación de la información.

Si queremos información sobre indicadores de reprobación en bachillerato podemos acudir a una base de datos especializada en educación y buscar todas las páginas que 
contengan la palabra bachillerato, las páginas que contengan la palabra reprobación, y las páginas que contengan la palabra indicadores; una mejor opción es buscar sólo aquellas páginas que contengan a la vez las palabras bachillerato, reprobación e indicadores.

La búsqueda de información no es un tema nuevo y durante la década de los 70 se le dio mucha importancia a la recuperación de la información, lo cual se debía a la complejidad de operación de las bases de datos accesibles en línea y a los costos asociados con la consulta de éstas. Actualmente al simplificarse la operación de las herramientas de búsqueda y reducirse los costos de operación, las instituciones educativas han dado menor importancia a la formación para la información, aunque desde la década de los 90, se inició una segunda ola de formación para la información que está presente en las principales instituciones educativas del mundo y que se conoce como Competencia en información o Alfabetización informativa.

En el pasado inmediato, las fuentes de información se ubicaban en las bibliotecas, actualmente, con la generalización de Internet y las facilidades de acceso a la Red, es posible contar con información distribuida en servidores de diferentes instituciones y países. Internet se ha convertido en el principal canal de información y consecuentemente, cada vez se incrementa el número de personas conectadas a la red y mayor el tiempo empleado en navegar, ya sea para acceder a las noticias, revisar el correo, ver videos, escuchar música, así como consultar enciclopedias, mapas, conversar por teléfono, participar en foros e interactuar con nuestros pares a través de redes, desde cualquier punto de la tierra; esto es, la red es mediadora en nuestro acceso e interpretación de la realidad (GRAU, 2008)

\section{La búsqueda de información y la capacidad cerebral}

Se dice que el cerebro humano se adapta a cada nuevo cambio y la disponibilidad de información en internet curiosamente contribuye a provocar un cambio al potenciar la capacidad humana como resultado de la construcción y operación de procesos de búsqueda. Una investigación reciente demostró que las personas que realizan búsquedas de información, construyendo sus estrategias de búsqueda, registran un incremento de la actividad en las regiones frontal y temporal del cerebro y en la llamada circunvolución 
singular, encargada de la toma de decisiones y de los procesos de razonamiento complejo. El grupo de investigación, dirigido por el científico Gary Small, encontró que el funcionamiento y rapidez de estos sectores del cerebro se duplicó en el grupo de voluntarios que manejaban los mecanismos y procedimientos de búsqueda de información en Internet (PIACENTE, 2008).

El hecho demostrado, de que el ejercicio intelectual aplicado en la búsqueda de información contribuye a la agilidad del cerebro y a optimizar su funcionamiento, aún no ha tenido un impacto en las instituciones de educación superior, no obstante contribuye a fortalecer la hipótesis de que la competencia en información ${ }^{2}$ tiene una correlación positiva con la capacidad innovadora. Por una parte, la disponibilidad de nuevos referentes aportados por la información, nos ayuda a realimentar nuestra práctica educativa, lo mismo que el efecto serendip o serendipia que hace referencia a hallazgos casuales o inesperados, que surgen en el proceso de búsqueda de información.

La serendipia surge cuando buscamos algún dato en específico y encontramos otros que no habíamos considerado y que eventualmente pueden ser de mayor utilidad. Este fenómeno se da cuando la mente está preparada para la búsqueda continua de significación en los hechos y en los datos que nos rodean. La serendipia ha acompañado a muchos de los inventos e innovaciones que se ha dado en la historia de la humanidad, por ejemplo, la leyenda de la manzana que cae y da origen a la teoría de la gravedad. Por ello es importante la búsqueda de información en los procesos de investigación e innovación y en la propia gestión de las instituciones educativas.

La información puede estar disponible localmente, aunque también es importante acercarse a fuentes externas que sirven como parámetros o referentes para orientar nuestras acciones o sugerir nuevas formas de enfrentar el quehacer académico en las instituciones educativas. En este contexto surgen nuevos términos y nuevas estrategias para abordar la búsqueda y aplicación de la información, tal es el caso de la vigilancia y la inteligencia colectiva.

\section{Información e innovación}

(C) Revista Digital de Biblioteconomia e Ciência da Informação,Campinas, v.7, n. 2, p. 166-184, jan./jun. 2010- ISSN: 1678-765X. 
Las organizaciones innovadoras exponen constantemente a sus miembros a nuevas ideas e innovaciones provenientes del exterior, por ello es importante que las organizaciones asuman que el conocimiento es un recurso distribuido ampliamente, que se comparte y se crea en forma colectiva y que, para aprovechar el volumen de información disponible en forma externa, se debe explorar y mantener fronteras porosas que absorban la información derivada de las prácticas innovadoras exitosas, así como de las lecciones aprendidas en los procesos de innovación (Cfr. CHUN, 1999, p. 173).

La innovación se asocia con la solución de un problema, entendido éste como una situación que dificulta la consecución de algún fin por lo que es necesario hallar los medios que nos permitan solucionarlo, atenuar o anular sus efectos, esto tiene significación por el hecho de que es posible conocer, estructurar y caracterizar el problema, consecuentemente también es posible identificar las necesidades de información para su solución, es decir, se hace uso de información y en forma colectiva se convierte en conocimiento que se aplica a mejorar los procesos, modos y formas de actuar inteligentemente frente a la realidad (MESÍAS RODRÍGUEZ Y ORTIZ, 2006).

En los procesos de innovación, las necesidades de información aparecen como lagunas en el conocimiento, en la comprensión o en las capacidades de la organización; se presentan como obstáculos para que la organización resuelva un problema o aproveche una oportunidad. Para abordar la búsqueda de información, se tiene que identificar y explicar detalladamente las necesidades que pertenecen a una situación problema. Definir y precisar el problema es parte del proceso de innovación y generalmente involucra a varios agentes que participan en diálogos y reflexiones para esclarecer la naturaleza y los límites de la situación, así como los objetivos y criterios de actuación en la experiencia innovadora (CFR. CHUN, 1999, p.168).

Adaptando el modelo de Eduardo Bueno Campos (1998), podemos percibir a la innovación como valor agregado al conocimiento, que se produce en tres grandes etapas en la cadena de la información, conforme a una secuencia cronológica. Primero, atiende la conversión de los datos en información. Segundo, convierte la información en conocimiento, en saber, lo cual es posible a través de determinado "proceso de aprendizaje. Y tercero, mediante la generalización se busca que el conocimiento se traduzca en "competencia esencial" o en la base sobre la que se pueda construir el "saber 
hacer". Para mejorar el bien o el servicio que es objeto de la actividad de la organización, esta competencia sólo es posible si se incorpora un "proceso de creación mental" determinado, integrando lógica y dinámicamente la información y el conocimiento con las ideas para la transformación de la realidad que caracterizan a la innovación (Véase figura 2).

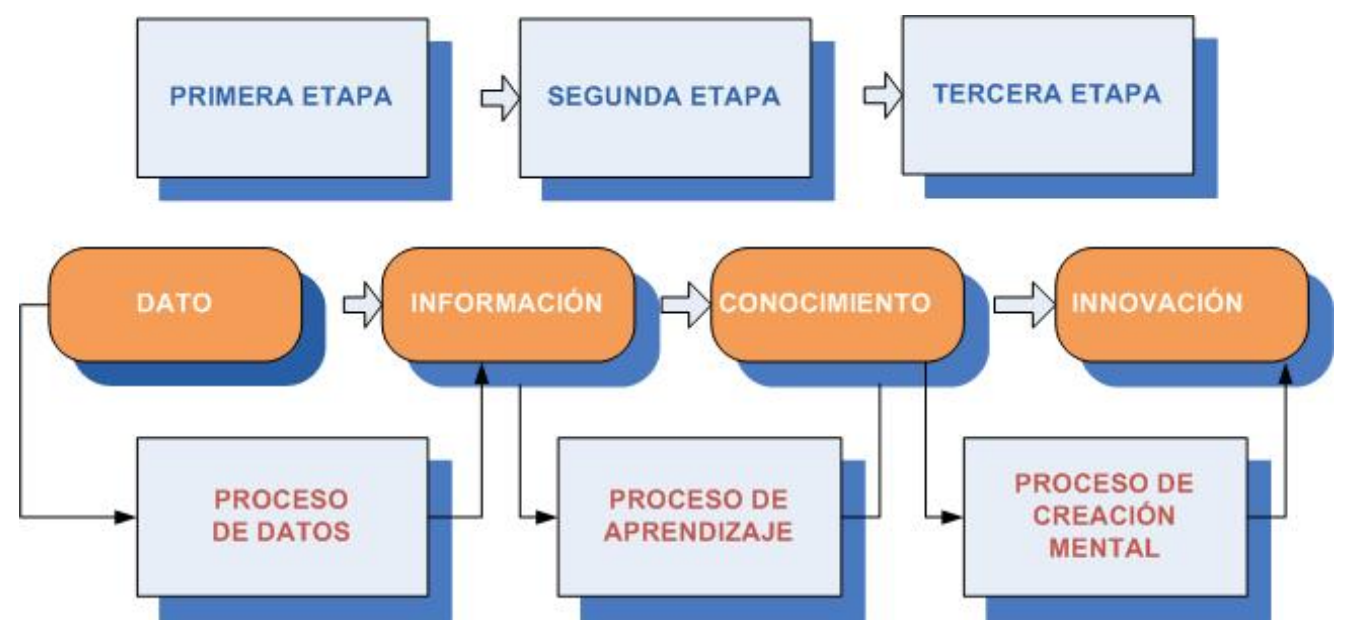

Figura 2. La innovación y valor agregado a la información

\section{Vigilancia e inteligencia}

La vigilancia del entorno es una práctica que se destina a la búsqueda de información externa, y en este proceso se encuentran "recursos" buenos, malos y regulares que después de su valoración y selección pueden transformarse en fuente de conocimiento para la organización. Los procesos de creación de conocimientos parten de la información y conocimientos ya disponibles en la organización, los que se enriquecen con la visión que aporta la información captada en el entorno. La inteligencia se apoya en la vigilancia para localizar toda aquella información susceptible de convertirse en valor para la organización, sea como innovación o como contribución a la solución de problemas (Cfr. MORCILLO, 2003).

La inteligencia se diferencia de la vigilancia por que trasciende al proceso de gestión de la información obtenida, la vigilancia se destina a la obtención de la información más relevante del entorno en congruencia con los objetivos de la organización, en tanto que la inteligencia atiende otros aspectos como la reelaboración y presentación de la información en una forma adecuada para la toma de decisión, así como el análisis de la 
evaluación de los resultados obtenidos de su aplicación. La vigilancia orienta las decisiones, en tanto que la inteligencia soporta la acción. (HIDALGO, LEÓN Y PAVÓN, 2002).

Para lograr un acceso óptimo a los recursos de información disponibles en Internet es necesario elaborar una estrategia de búsqueda, lo que implica seleccionar las palabras clave que mejor representan las necesidades de información e identificar los sinónimos y equivalentes, tanto en español como en otros idiomas. Si el buscador lo permite se deberán definir y precisar las relaciones lógicas entre términos usando los operadores booleanos por ello es importante conocer los aspectos básicos de operación de las herramientas de búsqueda y del propio proceso de búsqueda y recuperación de información.

\section{Tipos de búsqueda}

La búsqueda en Internet puede tener diferentes modalidades, cada una de éstas tiene sentido en tanto que nos dan la posibilidad de poder decidir en la extensión o la precisión en la recuperación de información. Esto es, si queremos tener más resultados o si lo que queremos es la información más precisa respecto a un tema de interés, lo cual implica que tendremos menos resultados pero estos serán más precisos en cuanto a nuestra necesidad específica de información. Típicamente podemos hablar de tres tipos de búsqueda de información: por palabra, por frase y búsqueda lógica o booleana, aunque otra opción es la búsqueda jerárquica, cuando se busca por temas en una estructura de índices.

\section{Qué es la búsqueda por palabras}

Consiste en suministrar una o más palabras clave, que tengan una relación directa con el tema de interés. El buscador toma en cuenta todas las palabras utilizadas y como resultado muestra una lista de todos aquellos documentos que contengan todos esos términos. Este tipo de búsqueda puede tener dos modalidades Búsqueda simple y búsqueda múltiple:

1. Búsqueda simple

(C) Revista Digital de Biblioteconomia e Ciência da Informação,Campinas, v.7, n. 2, p. 166-184, jan./jun. 2010- ISSN: 1678-765X. 
Aquella en la que se emplea una sola palabra de búsqueda, como por ejemplo, aprendizaje. Esta proposición nos arroja una lista de documentos que contienen en alguna de las partes 'del texto dicha palabra. El uso de palabras simples contribuye a lograr un mayor índice de recuperación pero reduce la precisión en los documentos recuperados.

\section{Búsqueda múltiple}

La proposición múltiple es de uso muy frecuente entre buscadores inexpertos, consiste en indicar más de un término simple, como por ejemplo búsqueda información revistas. Esta proposición, de tres palabras yuxtapuestas recupera una lista de aquellos documentos que contengan las palabras referidas sin importar su orden y posición. Su ventaja es que puede lograr una respuesta exhaustiva pero con un alto riesgo de recuperar documentos no deseados, en el mejor de los caso, recupera documentos sobre "búsqueda de información en revistas”, además de “revistas de búsqueda de información” e “información de búsqueda de revistas”, los cuales como se puede observar aunque tienen cierta similitud, expresan diferentes significados, en el peor de los casos recupera documentos sin que estos tengan una relación directa entre sí ni con el tema de interés, aunque cumplen la condición de incluir las palabras indicadas éstas se encuentran en distintos párrafos o páginas del documento y sin ningún orden de secuencia.

\section{Qué es la búsqueda por frase}

Si deseamos buscar, aquellos documentos que contengan de manera coincidente una secuencia dada de palabras, podemos utilizar la búsqueda por frase, delimitando entre comillas la secuencia de palabras que nos interesa encontrar. En la gran mayoría de las herramientas de búsqueda en Internet se dispone de una opción en la caja de búsqueda para incorporar la proposición de búsqueda por frase sin tener que utilizar el entrecomillado o algún otro carácter delimitador.

Esta modalidad de búsqueda permite expresar de manera precisa el tema de interés y resulta de mucha utilidad cuando se tiene poca experiencia en el diseño de estrategias de búsqueda de información. Se expresa como un argumento que se construye con más de una palabra en una secuencia dada. Por ejemplo, si se utiliza una frase encerrada entre 
comillas, el dispositivo de búsqueda recupera y muestra sólo aquellos documentos que contengan dicha frase, siempre que estén en el mismo orden que se indica. La respuesta puede variar de un buscador a otro pero, en todos los casos, aportan una respuesta más precisa que la búsqueda por palabras, no obstante, está latente el riesgo de exclusión involuntaria de documentos. Si utilizamos como argumento de búsqueda la frase “planeación curricular” estaríamos dejando fuera los documentos referidos por la frase “planificación curricular”, esto es, se excluyen de manera inadvertida los documentos referidos al mismo concepto, pero que emplean otros sinónimos o variantes ortográficas.

\section{¿Qué es una búsqueda booleana?}

La búsqueda booleana es una modalidad que permite formular búsquedas de información con un alto nivel de precisión, lo cual se logra mediante una ecuación lógica o yuxtaposición de palabras clave y utilizando los operadores booleanos OR, AND y NOT. La búsqueda booleana se emplea para decidir la relación deseada entre extensión y precisión en la recuperación de información, es particularmente útil cuando se desea expandir o limitar la búsqueda.

Los operadores booleanos permiten combinar palabras clave y/o frases para tener un mayor control sobre la operación de búsqueda, de este modo es posible la definición de conjuntos de búsqueda por intersección, disyunción, o exclusión usando los operadores AND, OR y NOT

- AND La ecuación de búsqueda TIC AND aprendizaje nos despliega únicamente aquellos documentos que contienen ambas palabras, es decir, la intersección del conjunto de documentos que contienen el primer término con el conjunto de documentos que contienen el segundo.

- OR: La ecuación de búsqueda redes OR comunidades despliega todos los documentos de la base de datos que contengan alguna de las palabras o todas las palabras relacionadas, es decir, la unión o suma lógica de aquellos documentos que contengan el primer término con el conjunto de documentos que contienen el segundo.

(c) Revista Digital de Biblioteconomia e Ciência da Informação,Campinas, v.7, n. 2, p. 166-184, jan./jun. 2010- ISSN: 1678-765X. 
- NOT: El operador de exclusión se antepone a una palabra para indicar que del conjunto de documentos que se propone recuperar se deberán excluir aquellos documentos no deseados. De esta manera, la ecuación de búsqueda Aprendizaje NOT Conductismo despliega el conjunto de documentos de la base de datos referidos al término Aprendizaje siempre y cuando estos no hagan referencia a la palabra Conductismo.

Nota: Algunos buscadores admiten una versión simplificada de los operadores booleanos AND y NOT para poder construir expresiones de búsqueda en forma más sencilla, aunque esto lo debemos confirmar en cada herramienta pues no todos lo permiten. El operador AND se indica con el signo “+”, en tanto que el operador de exclusión NOT se indica con el signo “-“, de esta manera, la ecuación de búsqueda +curriculum +matemáticas +educación -ingeniería, indica que se habrán de recuperar los documentos que contengan las palabras curriculum, matemáticas y educación pero sólo aquéllos que no estén referidos a ingeniería.

En la construcción de búsquedas booleanas es importante el orden de secuencia de los operadores, pero se debe tener presente que cada herramienta de búsqueda tiene su propia sintaxis e incorpora formularios, o cajas de búsqueda, donde se establecen valores por omisión u operadores implícitos y se dispone de espacios para precisar las operaciones lógicas de una manera sencilla.

\section{Herramientas de Búsqueda (Buscadores)}

Los primeros buscadores empleaban algoritmos para localizar datos almacenados en una base de datos local. La indexación de los datos aceleró y dio mayor precisión al proceso de búsqueda. Inicialmente se acudía al archivo invertido para posibilitar la recuperación de datos bibliográficos. Así surgieron los primeros motores de búsqueda, que operaban exclusivamente con datos textuales y tenían que ser alimentados por el operador del sistema. Las bases de datos distribuidas e Internet, hicieron posible la creación de un nuevo tipo de buscadores, ahora capaces de explorar e indexar los contenidos almacenados en servidores, permitir la búsqueda por palabras clave o por categorías

(C) Revista Digital de Biblioteconomia e Ciência da Informação,Campinas, v.7, n. 2, p. 166-184, jan./jun. 2010- ISSN: 1678-765X. 
temáticas y desplegar el resultado como un lista de documentos que incluyen las palabras clave utilizadas en la búsqueda. (VILLEGAS, 2007)

\section{Los motores de búsqueda o search engines}

Dispositivos de búsqueda diseñados para generar de manera automatizada, los índices de la información distribuida en la red y almacenarla en una base de datos propia. Podemos describirlos como sitios web diseñados para ayudar a los usuarios a encontrar la información que necesitan de manera sencilla, fácil y rápidamente. Se emplean cuando se tiene bien definida la necesidad de información y se han identificado de manera precisa las palabras que pueden contener los documentos relevantes, y cuando se quiere obtener el mayor número de documentos posibles referidos a un tema específico.

Un motor de búsqueda tiene 5 componentes:

1. El robot o araña que recorre la red para localizar documentos y que genera una base de datos textual con la descripción individual y complementada con la dirección de los ítems.

2. Un sistema de indización automática, según distintos criterios, ya sea de texto completo, texto parcial cuando se basa en el resumen o utilizando las etiquetas propias del lenguaje html (meta etiquetas).

3. Un dispositivo de búsqueda, una aplicación basada en algoritmos de búsqueda de cadenas de caracteres en una base de datos y presentación de datos en pantalla.

4. Un sistema de interrogación o “Query”: consiste de un lenguaje de consulta y una serie de procedimientos y sintaxis para precisar la cobertura, extensión y precisión en la búsqueda de información.

5. Un interface: que está representada por la caja de búsqueda y las páginas de presentación de los resultados.

Los motores de búsqueda emplean una aplicación que recorre la red para localizar documentos, que indiza e incorpora en una base de datos. Esta base de datos es interrogada por los usuarios a través de una caja de búsqueda que permite comparar la información disponible en la base de datos y la expresada por el usuario como su

(C) Revista Digital de Biblioteconomia e Ciência da Informação,Campinas, v.7, n. 2, p. 166-184, jan./jun. 2010- ISSN: 1678-765X. 
necesidad de información, devuelve como resultado un conjunto de documentos que contienen la información pertinente a las necesidades planteadas.

La forma de búsqueda en estos sistemas es a través de palabras clave introducidas en la caja de búsqueda, la mayoría de los buscadores están diseñados para posibilitar dos tipos de búsqueda: búsquedas simples y avanzadas. Las búsquedas suelen ser más exhaustivas que en los directorios, pero cuando no se saben utilizar devuelven resultados con mucho "ruido". Son útiles para responder a necesidades concretas de información. Ejemplos de motores: Google: (http://www.google.com); Altavista: (http://www.altavista.com/): Bing: (http://www.bing.com/?cc=es)

\section{Directorios:}

Un directorio es un índice temático que organiza la información a través de temas y subtemas, su operación se asocia a dispositivos de búsqueda en los que la información se encuentra agrupada en categorías o clases. Esta clasificación se hace generalmente de manera manual por un equipo de personas que se encarga de analizar cada sitio web y ubicarlo en alguna de las categorías temáticas previamente definidas. En algunos casos es el propio diseñador del sitio web quien se encarga de esta operación en el momento de incorporar los datos descriptivos de la página y su dirección a la base de datos.

Son de mucha utilidad cuando no se tiene una idea muy precisa de lo que se quiere, pero sí del tema o categoría en el que se agrupa. Este tipo de herramienta permite iniciar la búsqueda de la información con un tema general y, mediante selección del usuario, pasar a otros temas más específicos. Podemos describirla como una página que contiene un grupo de temas generales, al seleccionar cualquiera de ellos se despliega otro grupo de temas más específico y de manera sucesiva continuamos ese proceso descendente hasta ubicar el tema de nuestro interés o se agoten las categorías temáticas. La consulta en directorios se le conoce como búsqueda jerárquica. Ejemplos de directorios Buscopio: (http://www.buscopio.net/esp/); Busco.com: (http://www.busco.com/); Yahoo: (http://search.yahoo.com/), Combina las características del motor y del directorio; Directorio de blogs: (http://bitacoras.org.es/)

(c) Revista Digital de Biblioteconomia e Ciência da Informação,Campinas, v.7, n. 2, p. 166-184, jan.jjun. 2010- ISSN: 1678-765X. 


\section{Meta-buscadores}

Además de directorios y motores de búsqueda, existen otras herramientas que permiten mayor cobertura en la búsqueda de información, estos no tienen una base de datos propietaria pues son dependientes de otros buscadores. Se les conoce como metabuscadores o buscadores en paralelo. Estos sistemas se encargan de gestionar la consulta en buscadores seleccionados, generalmente respetan el formato original de los buscadores pero otros, además de incorporar otras facilidades, tienen una presentación uniforme, como es el caso de Copernic.

Se acude a los meta-buscadores cuando los resultados de una búsqueda en otras herramientas han sido muy bajos, cuando se trata de un tema poco común, o cuando se requiere de información exhaustiva sobre un tema en particular. Los meta-buscadores ofrecen detalles de las respuestas de cada uno de los servicios, o bien el listado completo de coincidencias. Ejemplos de metabuscadores: Dogpile (http://www.dogpile.com/); Metacrawler: (http://www.metacrawler.com/); Ixquick: (http://www.ixquick.com/); Copernic Search: (http://www.copernic.com/en/products/desktop-search/index.html) Este buscador requiere bajar algunas rutinas al disco duro.

\section{Motores de búsqueda de información académica}

La necesidad de contar con herramientas para la búsqueda de información académica motivó que la Editorial Elsevier desarrollara un sistema para indizar páginas de manera automática, tal como lo hacen los motores convencionales, pero con la capacidad de filtrar la información de manera adecuada y confiable para responder a los estrictos criterios académicos. Ese buscador se llamó Scirus (http://www.scirus.com/), su éxito motivó que Google implementara unos pocos años después Google Académico: (http://scholar.google.com.mx/)). Eric: (http://www.eric.ed.gov/) Más que un buscador, se trata de una biblioteca digital en educación. Otra herramienta de interés académico es SCITOPIA: (http://www.scitopia.org). La perspectiva que combina rigor académico y máxima capacidad de recuperación corresponde a Scirus (CODINA, 2007).

\section{Comentario final y conclusiones}

(C) Revista Digital de Biblioteconomia e Ciência da Informação,Campinas, v.7, n. 2, p. 166-184, jan./jun. 2010- ISSN: 1678-765X. 
En el ámbito de la innovación educativa, la información tiene la doble función de soporte e insumo en los procesos de innovación, El agente innovador está en permanente interactividad con la información local, en interacción continua con otros agentes, y es vigía para detectar la información externa que es de utilidad. La búsqueda de información ha sido y será una forma de poner en contacto al usuario de la información con los recursos pertinentes disponibles, por ello se han diseñado dispositivos de software para atender diferentes tipos de necesidades o características de los recursos de información. La mayor ventaja que se puede obtener de estas herramientas de búsqueda no depende propiamente de su diseño o de la infraestructura tecnológica a la que se tenga acceso, sino de la capacidad de articular las herramientas de búsqueda con una clara definición de necesidades e intereses del usuario.

Por otra parte, debemos ser cuidadosos pues si bien Internet nos da acceso a datos, con frecuencia son de baja calidad, y sin ningún tipo de control sobre su veracidad, por ello la importancia de los buscadores académicos. La confusión detrás del término "sociedad del conocimiento" es asumir que conocimiento es información, pero la información, por sí misma, no tiene efecto alguno sobre la realidad si no se procesa y se convierte en conocimiento. La innovación se sustenta en información sobre el problema o la realidad que se quiere transformar, pero no de la colecta y almacenamiento sino de su análisis y proceso, para su conversión en conocimiento útil. La información sobre el problema se complementa con la información sobre posibles soluciones y a partir de este insumo surgen nuevas ideas creativas que dan valor agregado al conocimiento y estimulan la innovación.

Aunque cada vez se dispone de mejores herramientas de búsqueda, ninguna sustituye la capacidad humana para establecer la pertinencia de los datos, su conversión en información y en conocimiento. También vale decir que ninguna máquina está hecha para los hallazgos inesperados que son parte de la agudeza humana para ver lo que pasa desapercibido para las herramientas de búsqueda y para las personas que no han desarrollado su competencia en información. Es esta capacidad la que nutre el quehacer de la innovación, por ello se propone la formación para la búsqueda de información en los procesos institucionales de desarrollo de la capacidad de innovación de los docentes.

(c) Revista Digital de Biblioteconomia e Ciência da Informação,Campinas, v.7, n. 2, p. 166-184, jan./jun. 2010- ISSN: 1678-765X. 
El objetivo de esta propuesta es motivar el interés y contribuir a desarrollar en los docentes, las habilidades para la búsqueda, selección y utilización de información y su conversión en conocimiento e innovación, por ello convendría establecer una evaluación diagnóstica que permita tener un panorama de las habilidades y actitudes hacia el manejo de la información y su impacto en la capacidad innovadora de los docentes, antes y después de formarse en las competencias en información. Se asume que los docentes interesados en la innovación educativa tendrán mejores posibilidades de éxito si se ocupan de mejorar sus capacidades y destrezas en la búsqueda, recuperación, registro y organización de información; en el marco de las actividades implicadas en el ciclo de generación del conocimiento y su conversión en innovación.

\section{Notas:}

1. En 2003 se estimaba que el volumen de información en Internet, accesible al público era de 167 terabytes en tanto que la información en Internet oculto era de 400 a 450 veces mayor, esto es entre 66,800 y 91,850 terabytes. Existían 2.9 millones de blogs activos, conteniendo alrededor de 81 GB de información. Fuente: UC Berkeley's School of Information Management and Systems (2003) How Much Information? Se estima que en 2003 circulaban en Internet diariamente 31 billones de e-mails Internet y se esperaba que esa cifra se duplicara en 2006 (Fuente: International Data Corporation (IDC). La extensión promedio de emails es alrededor de 59 kilobytes, y el flujo anual de emails en la web fue de 667,585 terabytes.

2. En artículos anteriores, disponibles en la red, se aborda el tema de las competencias en información y su relación con la innovación, así como el problema de la información: 1) "Relación entre competencia en información e innovación educativa”. Revista Brasileira de Biblioteconomia e Documentação, Nova Série, São Paulo, v.4, n.2, p. 4-14, jul./dez. 2008. En: (http://www.febab.org.br/rbbd/ojs.1.1/index.php/rbbd/article/viewPDFInterstitial/110/124); 2) "El problema de la información en el contexto de la educación superior”. Forinf@ Online: Revista iberoamericana sobre usuarios de la información, ISSN 1695-6869, №. 26, 2004, pags. 9-18. En: (http://lemi.uc3m.es/forinf@/IMG/pdf/info_26.pdf); 3) “Normas de competencia en información”. BiD: Textos universitaris de biblioteconomia i 
documentació, ISSN 1575-5886, No. 11, 2003. En:

http://www2.ub.es/bid/consulta_articulos.php?fichero=11angul2.htm)

\section{Referencias}

Bueno Campos, Eduardo (1998) Agregar valor a la información y al conocimiento. En: http://www.madrimasd.org/informacionidi/revistas/Numero1/cara1.asp

Codina. Lluís (2007) Motores de búsqueda de información científica y académica. Hipertext.net, núm. 5, En: < http://www.hipertext.net > [Consulta: 17/05/109]. ISSN 1695-5498. http://www.hipertext.net/web/pag273.htm ]

Delclós, Tomás (2007) La búsqueda automatizada no ha logrado excluir el factor humano del experto, En:

http://www.elpais.com/articulo/red/busqueda/automatizada/ha/logrado/excluir/factor/hum ano/experto/elpeputeccib/20070517elpcibenr_2/Tes

Chun Wei Choo (1999) La organización inteligente: el empleo de la información para dar significado, crear conocimiento y tomar decisiones.

Fuentes Agustí, Marta (2001) Naufragar en Internet. Estrategias de búsqueda de información en redes telemáticas. Virtual Educa 2001 (27-29 de junio de 2001) En: http://www.uoc.edu/web/esp/art/uoc/0109037/fuentes.html

Grau, Abel (2008) Internet cambia la forma de leer... ¿y de pensar? Sala de Prensa: Web para profesionales de la comunicación iberoamericanos. Noviembre. Año X, Vol. 4, núm. 109, en: http://www.saladeprensa.org/art786.htm

Hidalgo, A., G. León y A. Pavón (2002) La gestión de la innovación y la tecnología en las organizaciones. Madrid: Pirámide.

Mesías Ratto, Rosa Victoria, Rosa Bertha Rodríguez Vera y Federico Ortiz Agurto (2006) Guía para el desarrollo de la capacidad de solución de problemas. En: http://www.scribd.com/doc/6403714/GUIA-PARA-EL-DESARROLLO-DE-LACAPACIDAD-PARA-LA-SOLUCION-DE-PROBLEMAS

Morcillo, Patricio (2003) Vigilancia e inteligencia competitiva: fundamentos e implicaciones. MadrI+D. n. 17 (junio)

http://www.madrimasd.org/revista/revista17/tribuna/tribuna1.asp

Piacente, Pablo (2008) La búsqueda de información en Internet estimula importantes funciones cerebrales (28 de Octubre de 2008). En: http://www.coguan.com/blog/labusqueda-de-informacion-en-internet-estimula-importantes-funciones-cerebrales/

Villegas Núñez, Paulo (2007) Motores de búsqueda: presente y retos. Boletín de la Sociedad de la información: tecnología e innovaciónhttp://sociedaddelainformacion.telefonica.es/jsp/articulos/detalle.jsp?elem=4643 


\section{Noel Angulo}

Instituto Politécnico Nacional. Licenciado em Biblioteconomia e Mestre em Metodología da Ciência. noangulo@hotmail.com

Recebido em: 05/08/2009

Aceito para publicação em: jul/2009 